\title{
A Remark on One Family of Iterative Formulas
}

\author{
Dragomir M. Simeunović
}

Abstract. In this paper we obtain one family of iterative formulas of the second order for finding zeros of a given function $F(x)$.

In this paper, starting from the Newton's iterative formula

$$
x_{k+1}=x_{k}-\frac{F\left(x_{k}\right)}{F^{\prime}\left(x_{k}\right)}, k=0,1,2, \ldots,
$$

which we write in the form

$$
x_{k+1}=x_{k}\left(1-\frac{F\left(x_{k}\right)}{x_{k} F^{\prime}\left(x_{k}\right)}\right), k=0,1,2, \ldots,
$$

we obtain a family of iterative formulas of the second order.

If, instead of

$$
1-\frac{F\left(x_{k}\right)}{F^{\prime}\left(x_{k}\right)}
$$

we put

$$
\left(1-\frac{F\left(x_{k}\right)}{s x_{k} F^{\prime}\left(x_{k}\right)}\right)^{s}
$$

in formula (2), where $s \neq 0$ is a real parameter, we get an iterative formula

$$
x_{k+1}=x_{k}\left(1-\frac{F\left(x_{k}\right)}{s x_{k} F^{\prime}\left(x_{k}\right)}\right)^{s}, k=0,1,2 \ldots
$$

The expression (3) represents one family of iterative formulas, i.e. one iterative method, of the second order.

If we now consider different real values for the parameter $s$, we obtain particular iterative formulas from (3).

For $s=1,(3)$ reduces to Newton's method (2).

For finding zeros of the polynomial

$$
P(x)=a_{0}+a_{1} x+a_{2} x^{2}+\cdots+a_{n} x^{n},
$$

2000 Mathematics Subject Classification. Primary: 65H05.

Key words and phrases. Iteration formulas, Approximate solutions of equations. 
of the degree $n$, we take $s=\frac{1}{n}$ if $n$ is odd. In this case, the formula (3) reduces to

$$
x_{k+1}=x_{k}\left(1-\frac{n P\left(x_{k}\right)}{x_{k} P^{\prime}\left(x_{k}\right)}\right)^{\frac{1}{n}}, k=0,1,2, \ldots
$$

If $n$ is even, then we take $s=\frac{1}{n-1}$. In that case, (3) becomes

$$
x_{k+1}=x_{k}\left(1-\frac{(n-1) P\left(x_{k}\right)}{x_{k} P^{\prime}\left(x_{k}\right)}\right)^{\frac{1}{n-1}}, k=0,1,2, \ldots
$$

The location of the zeros of the polynomial (4) in the complex plane, depending on its coefficients $a_{k}$, where $k=0,1,2, n$, were studied by many authors (see, e.g. [1]). Here we cite two results due to Cauchy [1, pp. 122123] and a result due to P. Montel [2] which are, respectively, as follows:

$\left(R_{1}\right) \quad$ All the zeros of the polynomial (4) lie in the circle

$$
|x| \leq r
$$

where $r$ is a positive root of the equation

$$
P_{1}(x)=\left|a_{n}\right| x^{n}-\left|a_{n-1}\right| x^{n-1}-\cdots-\left|a_{1}\right| x-\left|a_{0}\right|=0 .
$$

$\left(R_{2}\right) \quad$ All the zeros of the polynomial (4) lie in the region

$$
|x|<1+A \text {, }
$$

where

$$
A=\max \left|\frac{a_{k}}{a_{n}}\right|, k=0,1,2, \ldots, n-1 .
$$

$\left(R_{3}\right) \quad$ All the zeros of the polynomial (4) lie in the region

$$
|x|<2 M,
$$

where

$$
M=\max \left|\frac{a_{n-k}}{a_{n}}\right|, k=1,2, \ldots, n .
$$

For determining the upper bound for the moduli of zeros of the polynomial (4), we can use the formula

$$
x_{k+1}=x_{k}\left(1-\frac{n P_{1}\left(x_{k}\right)}{x_{k} P_{1}^{\prime}\left(x_{k}\right)}\right)^{\frac{1}{n}}, k=0,1,2, \ldots
$$

The method (13), for $x_{0}>1+A$, converges monotonically to $r$.

Example. We determine the upper bound for the moduli of zeros of the polynomial

$$
P(x)=x^{5}-5 x+22
$$

using the formula (13), where

$$
P_{1}(x)=x^{5}-5 x-22,
$$

for $x_{0}>1+A$. 
Taking $x_{0}=30$, we obtain

Newton's method

Method using formula (13)

$$
\begin{aligned}
x_{k+1} & =x_{k}-\frac{P_{1}\left(x_{n}\right)}{P_{1}^{\prime}\left(x_{k}\right)} \\
x_{0} & =30 \\
x_{1} & =24.00003506 \\
\vdots & \\
x_{5} & =7.866439195 \\
\vdots & \\
x_{10} & =2.675267821 \\
\vdots & \\
x_{14} & =2.000013231 \\
x_{15} & =2.000000000
\end{aligned}
$$$$
x_{k+1}=x_{k}\left(1-\frac{5 P_{1}\left(x_{k}\right)}{x_{k} P_{1}^{\prime}\left(x_{k}\right)}\right)^{\frac{1}{5}}
$$$$
x_{0}=30
$$$$
x_{1}=2.69437266
$$$$
x_{2}=2.017339895
$$$$
x_{3}=2.000019658
$$$$
x_{4}=2.000000000
$$

\section{REFERENCES}

[1] M. Marden, Geometry of Polynomials, Amer. Math. Soc. Providence, R.I. 2005.

[2] P. Montel, Sur quelques limites pour les modules des zéros des polynômes, Comment. Math. Helv., Vol. 7, 1934-35, 178-200.

Dragomir M. Simeunović Mike Alasa 8 11000 BELGRADE

SERBia 
